\title{
DIRECT CALCULATION OF THE SURFACE-TO-VOLUME RATIO FOR HUMAN CANCELLOUS BONE
}

\author{
D. P. Fyhrie, ${ }^{*}$ N. L. Fazzalari, ${ }^{*} \dagger$ R. Goulet $\ddagger$ and S. A. Goldstein $\ddagger$ \\ ${ }^{*}$ Breech Research Laboratory, Bone and Joint Center Henry Ford Hospital, Detroit, MI 48202. U.S.A; \\ $\ddagger$ The Orthopaedic Research Laboratories Orthopaedic Surgery, University of Michigan, Ann Arbor. \\ Michigan, U.S.A
}

\begin{abstract}
There are many diseases which cause detrimental changes in the trabecular structure of cancellous bone, leading to mechanical failure of the tissue. One approach to understanding the mechanisms of these diseases is to create idealized models that recreate the morphology of the tissue. This paper presents a partial development of such a model. Further histological methods must be developed before a complete definition of morphologically valid models is possible.

In a histological section of cancellous bone, the orientation and length of the trabecular surfaces determine how a line drawn across the bone section will intersect the bone-marrow interface. The distribution of the average length between intersections for a set of parallel lines is defined as the mean intercept length distribution. In this paper, the average surface morphology and volume of the average structure of cancellous bone is determined from an examination of the mean intercept length. The average structure of cancellous bone contains a repeated structural element (SE). As a result, the basic bone structure is analogous to a brick wall made from many similar bricks. For a group of 107 specimens, a strong relationship between structural element volume (SE.V) and bone volume fraction (BV/TV) is demonstrated, $S E . V=0.017 \kappa(B V / T V)^{-2.05} \mathrm{~mm}^{3}, R^{2}=0.93$, with $\kappa$ a model-dependent constant. For the same specimens, the structural element surface (SE.S) showed the relationship, SE.S $=0.144 \kappa(\mathrm{BV} / \mathrm{TV}){ }^{1.35}$, $R^{2}=0.92$. As a result of the inverse square dependence of structural element volume on bone volume fraction, it is predicted that cancellous bone strength is inversely proportional to structural element volume.
\end{abstract}

\section{INTRODUCTION}

The determination of cancellous bone strength, stiffness and biological dynamics has long been an important area of investigation due to the many diseases and conditions that cause the mechanical failure of cancellous bone. An area of intense investigation has been the relationship between the tissue microstructure of the bone and its mechanical properties. One goal has been to understand how changes in the normal microstructure and mass of the trabeculae can cause detrimental changes in the mechanical properties of cancellous bone, perhaps leading to overt failure of the bone to provide support for the body (e.g. osteoporosis). Fundamental to these investigations are the methods of histomorphometry. Taken as a whole, these methods provide a means to assess quantitatively the static structure of the hard tissue, the cellular dynamics and also the connection between cellular dynamics and the transformation of hard-tissue structure (Parfitt et al., 1983; Weibel, 1979). The purpose of this work was to develop a generalized three-dimensional model for cancellous bone.

First received 12 February 1991; accepted 8 December 1992.

Address correspondence to: David P. Fyhrie, Ph.D., Head, Section of Biomechanics, Breech Research Laboratory, Bone and Joint Center, Menry Ford Hospital, Detroit, MI 48202, U.S.A

†Permanent address: Institute of Medical and Veterinary Science, Adelaide, Australia.
The properties of the model as a function of bone volume fraction were determined experimentally.

One measure of the anisotropy and microstructure of cancellous bone is the mean intercept length (Harrigan and Mann, 1983; Whitehouse, 1974) or its inverse (Raux et al., 1975). The mean intercept length has been widely used in the field of biomechanics as a means for predicting cancellous bone stiffness and strength using both empirical (Goldstein et al., 1990; Goulet et al., 1988; Snyder and Hayes, 1990; Snyder et al., 1989; Turner et al., 1988) and highly theoretical approaches (Cowin, 1985, 1989). It is used, in part, because it is fairly easy to calculate using a grid of parallel lines placed over a histological section of bone. Using the grid, the mean intercept length is the average distance between the intersections of the grid lines with the bone-marrow interface.

The motivation for using the mean intercept length to characterize cancellous bone microstructure is that the mechanical properties of cancellous bone are directly related to its microstructure. If the mean intercept length uniquely characterizes cancellous bone microstructure, then bone stiffness and strength can be largely determined using histomorphometric methods. Unfortunately, as observed originally by Hilliard (1962), Philofsky and Hilliard (1969) and recently discussed by Odgaard et al. (1990), the mean intercept length does not uniquely determine microstructure because it is only dependent upon the length and orientation of the bone-marrow interface, rather than upon the position of the parts of the interface. In fact, if the bone marrow interface is cut into small 
parts and arbitrarily rearranged within the original area without rotating the pieces, the mean intercept length for the cut and redistributed pattern will be identical to the original mean intercept distribution. It is, therefore, inherently impossible for the mean intercept length to completely characterize the structure of cancellous bone. However, the mean intercept length does strongly restrict the class of microstructure to which the trabeculae can belong. In particular, it detcrmines the length of the bone-marrow interface and the possible slopes (orientations) of all of the parts of the bone-marrow interface. The work in this paper determines the nominal bone-marrow interface shape associated with the ellipsoidal mean intercept length distribution measured experimentally from human cancellous bone.

\section{THEORETICAL BACKGROUND}

One definition of the mean intercept length measure for a two-dimensional histological cross-section of cancellous bone $[H(\theta)]$ is (Harrigan and Mann, 1983)

$$
H(\theta)=\frac{1}{P_{l}(\theta)},
$$

where $P_{l}(\theta)$ is the number of intersections which a grid of parallel lines makes with the bone surface per total length of grid line and $H(\theta)$ is the average distance between the intersections. To calculate $P_{l}(\theta)$ directly, a grid of parallel lines is oriented over an image of the bone surface at the angle $\theta$, and the number of intersections between the lines and the bone-marrow interface is recorded. This number is then divided by the total length of line in the parallel array.

Another form of the mean intercept length has been used (Goulet et al., in preparation; Snyder et al., 1990; Whitehouse, 1974), which is defined as

$$
L(\theta)=\frac{2(\mathrm{BA} / \mathrm{TA})}{P_{l}(\theta)},
$$

for a two-dimensional histological section. Here, $P_{l}$ is as above and BA/TA is the area fraction of the hard tissue in the section. The reason for the existence of these two definitions is that the first was originally intended for use with grainy materials, such as steel, that are made of only one material, and the second was intended for use with materials, such as cancellous bone, that have more than one phase (Underwood, 1970). In both instances, the intent is to obtain an estimate of the size of the elements, either the grain size in the first case, or the size of one of the phases in the second case. In this paper the first definition of the mean intercept length $(H)$ was used for mathematical convenience since it is not theoretically dependent on the area fraction of the hard tissue (BA/TA) which, in three dimensions is equivalent to not being dependent on the bone volume fraction, BV/TV (Weibel, 1979).

For cancellous bone it has been shown (Harrigan and Mann, 1983; Stone et al., 1984; Whitehouse, 1974) that the mean intercept length of bone, when plotted as a polar plot, very nearly forms an exact ellipsoid. Mathematically (two dimensions), this is

$$
\begin{aligned}
\frac{1}{H^{2}(\theta)} & =P_{l}^{2}(\theta) \\
& =\left(\frac{\cos \theta}{H_{1}}\right)^{2}+\left(\frac{\sin \theta}{H_{2}}\right)^{2},
\end{aligned}
$$

where $H_{1}, H_{2}$ are the lengths of the major and minor axes of the ellipse and the angle $\theta$ is measured counterclockwise from the $X$-axis (Fig. 1). Note that (1) the quantities $H_{i}, i=1,2$, are the same as the eigenvalues of the 'fabric tensor' defined by Cowin (1989), and that (2) this equation assumes that the ellipse has its axes aligned with the coordinate $(X-Y)$ axes. It is significant to note that the mean intercept length does not directly represent the shape of any part of cancellous bone structure.

\section{Calculation of an equivalent bone-marrow interface}

Hilliard (1962) and Philofsky and Hilliard (1969) have shown that a convex body with an arbitrary, smooth distribution of $P_{l}(\theta)$ can be determined using the mathematical recipe

$$
L_{A}(\theta)=(1 / 2)\left[\mathrm{d}^{2} P_{l}(\omega) / \mathrm{d} \omega^{2}+P_{l}(\omega)\right]_{\omega=\theta+\pi / 2},
$$

and

$$
\begin{array}{ll}
x_{A}(\gamma)=\int_{0}^{\gamma} L_{A}(\theta) \cos \theta \mathrm{d} \theta, & 0 \leqslant \gamma<\pi, \\
y_{A}(\gamma)=\int_{0}^{\gamma} L_{A}(\theta) \sin \theta \mathrm{d} \theta, & 0 \leqslant \gamma<\pi,
\end{array}
$$

where $x_{A}(\gamma)$ and $y_{A}(\gamma)$ are parametric equations in angle $\gamma$ describing the perimeter per unit area of a convex shape with the mean intercept length distribution, $1 / P_{l}(\theta)$

For this paper the convex shape with the same mean intercept length ellipse as real bone was determined. Consequently, the quantity $L_{A}(\theta)$ is the length
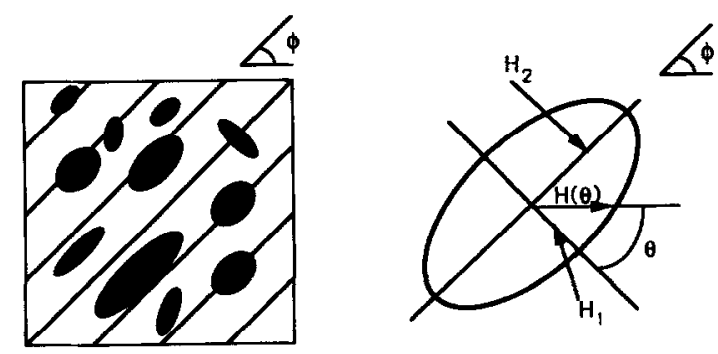

$$
P_{1}(\theta)^{2}=[1 / H(\theta)]^{2}=\left[\cos (\theta) / H_{1}\right]^{2}+\left[\sin (\theta) / H_{2}\right]^{2}
$$

Fig. 1. The mean intercept distance $H(\theta)$ is calculated by counting the intercepts as a function of angle $\left[P_{l}(\theta)\right]$ and inverting. For bone, the correlation of the intercept counts to an ellipse is very strong. In principal axes, the ellipse has the form shown in the figure. 
distribution of the trabecular bone. In terms of quantities identifiable from a histological section, $L_{A}$ is the length of bone-marrow interface per unit total tissue area that has inclination $\theta$ with respect to the global coordinate axes. The angle $\theta$ was again measured counterclockwise from the $X$-axis. This definition of the length distribution $\left(L_{A}\right)$ is valid for the interface between constituents of any two-phase material.

The physical position of the perimeter is calculated using

$$
\begin{aligned}
& x=x_{A} \times A, \\
& y=y_{A} \times A,
\end{aligned}
$$

where $A$ is the area of the cross-section that contains the bone-marrow interface.

Since $L_{A}$ is directly derivable from the value of $P_{I}$ and its second derivative, for any $P_{l}$ that is smooth, $L_{A}$ is smooth. Physically, the smoothness of $P_{l}$ implies that at least some of the bone-marrow interface is pointing in all possible directions. This is reasonable from the examination of a real bone section where at least part of the interface is parallel to any arbitrarily chosen line. Certainly, the second derivative of $P_{l}$ exists if the approximation to the mean intercept distribution is elliptical.

Since the $P_{l}$ measurement contains no information about the placement of the pieces of the interface (see the Introduction), neither can $L_{A} . L_{A}$ does, however, represent the totality of the interface by indicating how much of it exists at each of the possible angles of inclination. The lack of information about placement of the parts of the interface in space is the source of the convex body assumption mentioned above. The integral equations (4) present one of the infinite methods by which a shape can be constructed from $L_{A}$. By their nature, these integral equations cause construction of a convex shape. This is not a limitation of the approach, but is one of its consequences.

The above equations describe $P_{l}, L_{A}$ and, consequently, $(x, y)$ for cancellous bone. In principle, it is possible to calculate the shape of a two-dimensional body for any smooth mean intercept length distribution using the above equations. Using the symbolic mathematics computer program MACSYMA (Symbolics, Inc.), it was possible to solve for the convex shape associated with the special case of the elliptical mean intercept length distribution that is common for cancellous bone [equation (3)]. The mathematical derivation (Appendix 1) gives the following result:

$$
\left(2 H_{1} x_{A}\right)^{2}+\left(2 H_{2} y_{A}\right)^{2}=1, \quad y_{A}>0,
$$

where all terms are as previously defined. The symbolic integration was tested by comparison to numerical integration of the equations for special cases.

If we recall, $x_{A}=x / A$, and $y_{A}=y / A$, then

$$
\left(\frac{2 H_{1} x}{A}\right)^{2}+\left(\frac{2 H_{2} y}{A}\right)^{2}=1, \quad y>0 .
$$

Both equations (6) and (7) form the top halves of ellipses because of the restriction on the values of $y_{A}$ and $y$. These semi-ellipses have the correct shape and length distribution such that, for any arbitrary area of interest, $A$, the length distribution of the semi-ellipse combined with the area $A$ has the identical mean intercept length distribution as the real bone cross section.

The radii of these semi-ellipses are $R_{1}=A /\left(2 H_{1}\right)$ and $R_{2}=A /\left(2 \mathrm{H}_{2}\right)$ (Fig. 2). The perimeter of the semiellipse, $L$, is approximately (Selby, 1971)

$$
\begin{aligned}
L & \approx \frac{1}{2}\left\{2 \pi\left(\frac{R_{1}^{2}+R_{2}^{2}}{2}\right)^{1 / 2}\right\}, \\
& \approx \frac{\pi}{2} A\left\{\frac{1}{2}\left(\frac{1}{H_{1}^{2}}+\frac{1}{H_{2}^{2}}\right)\right\}^{1 / 2},
\end{aligned}
$$

where the shape of the area $A$ and its magnitude are completely arbitrary. An approximation was used to estimate the perimeter length of the ellipse because the exact form is an elliptic integral.

The total trabecular bone-marrow interface length (Tb.L) per unit total area of bone (TA) was calculated immediately as

$$
\mathrm{Tb} . \mathrm{L} / \mathrm{TA}=\mathrm{L} / \mathrm{A} \approx \frac{\pi}{2}\left\{\frac{1}{2}\left(\frac{1}{H_{1}^{2}}+\frac{1}{H_{2}^{2}}\right)\right\}^{1 / 2} .
$$

This equation gives the total bone-marrow interface length per unit tissue area for any arbitrary choice of total tissue area TA.

\section{Introduction of the two-dimensional structural element}

Equations (6) and (7) define semi-ellipses which have the same length distribution and mean intercept length distribution as a two-dimensional histological section of real cancellous bone for an arbitrary tissue area, TA. Equation (9) is the average bone-marrow interface per tissue area, TA. This area includes both the hard-tissue area and marrow area over which the bone-marrow interface is distributed. The combination of the two areas is essential to understanding cancellous bone microstructure because it is the combination of bone and marrow which creates the interface. A fundamental property of the mean intercept length is that it provides no information permitting distinction between the hard tissue and marrow components of the bone. This is a consequence of it being a measurement of the interface distribution rather than of any property of the two phases of the structure. Therefore, it was not unexpected that the important area (TA) combines both marrow and hard-tissue areas in an undifferentiated manner.

When interpreting the above results as representative of a repeating structural element, the notation for the tissue area, ' $T A=A$ ', is renamed 'SE.A $=\mathbf{A}$ ', consistent with the standard for histomorphometry 


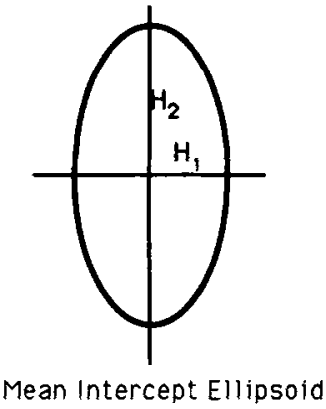

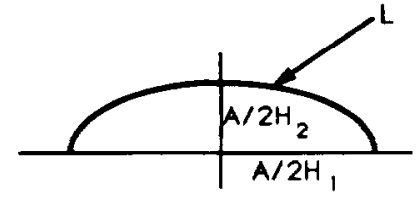

Semi-ellipse of Bone-marrow Interface

Fig. 2. The relationship between the mean intercept ellipsoid and the calculated semi-ellipse of the bone-marrow interface.

(Parfitt et al., 1987). Similarly, the consistent notation for the distributed length, 'Tb.L $=L$ ' is 'SE.L.' In both cases, the letters ' $S E$ ' indicate that the area is associated with a fundamental, repeating structural element. Note that the ratio SE.L/SE.A is identical to Tb.L/TA and that the ratios TA/SE.A =Tb.L/SE.L measure the number of structural elements that appear on a section of size TA.

To understand the above results in terms of a repeated structural element for cancellous bone, an assumption must be made about how the structural element area changes as a function of the mean intercept length. The definition of these structural elements was based on the choice of their shape and size. For instance, one possible definition of the SE is a rectangle of side ratio $H_{1} / H_{2}$ with area SE.A $=H_{1} \times H_{2}$. Several examples of the consequences of this type of assumption were examined (Fig. 3).

It is significant that it is not necessary to know the actual distribution of hard tissue in the structural element area to obtain an exact representation for SE.L/SE.A. Consequently, the bone area fraction, BA/TA, of the tissue is not restricted by the results in Fig. 3. To reproduce BA/TA, the perimeter of the semi-ellipse must be distributed in the area SE.A to divide the plane into two phases, hard tissue and marrow, with the ratio of hard-tissue area to SE.A equal to the BA/TA of the real bone cross section. The mean intercept length provides no information on how this might be done correctly and the development of techniques to do this are beyond the scope of this work.

\section{Generalization of the two-dimensional structural element}

For every proposed two-dimensional structural element shape (Fig. 3), the dependence of SE.A on the $H_{i}$ was

$$
\text { SE.A }=\kappa H_{1} H_{2} \text {, }
$$

where $\kappa$ is a constant characteristic of a particular structural element. Recognizing this regularity in the models, the generalized form of the two-dimensional structural element is [using equations (9) and (10)]

$$
\begin{aligned}
\frac{\text { SE.L }}{\kappa} & \approx \frac{\pi}{2}\left\{\frac{1}{2}\left(H_{1}^{2}+H_{2}^{2}\right)\right\}^{1 / 2}, \\
\frac{\text { SE.A }}{\kappa} & =H_{1} H_{2}, \\
\text { SE.L } / \text { SE.A } & \approx \frac{\pi}{2}\left\{\frac{1}{2}\left(\frac{1}{H_{1}^{2}}+\frac{1}{H_{2}^{2}}\right)\right\}^{1 / 2} .
\end{aligned}
$$

These equations and equation (8) form the basis for developing an approximation for the three-dimensional properties of the structural element.

\section{THE THREE-DIMENSIONAL STRUCTURAL ELEMENT}

In three dimensions, the mean intercept length ellipsoid for cancellous bone in principal coordinates is

$$
\frac{1}{H^{2}(l, m, n)}=\left(l / H_{1}\right)^{2}+\left(m / H_{2}\right)^{2}+\left(n / H_{3}\right)^{2},
$$

where the $H_{i}$ are the principal radii and $(l, m, n)$ are direction cosines.

For prediction of the distribution of bone surfaces in three dimensions from the three-dimensional mean intercept ellipsoid, first note that the two-dimensional equations for line distribution [Equation (8)] are valid for cross sections cut at any orientation through the three-dimensional structure. In particular, the two-dimensional equations are valid for the threedimensional principal axes of the structure. For each of the mutually perpendicular principal axes of the structure that would be predicted by a three-dimensional analysis, the mean intercept distribution predicted by the two-dimensional analysis would be an ellipse. The three-dimensional surface most suggested by these three mutually perpendicular ellipses is an ellipsoid. The surface distribution for the cancellous bone is reproduced by an ellipsoid with principal axes of magnitudes

$$
R_{i}=\frac{3}{4} H_{i}, \quad i=1,2,3,
$$

as demonstrated in Appendix 3. 


$$
\begin{aligned}
& \text { SE.AmK } H_{1} H_{2} \\
& \text { SE.L } \approx 2 \times\left\{(1 / 2)\left(H_{1}^{2}+H_{2}^{2}\right)\right\}
\end{aligned}
$$
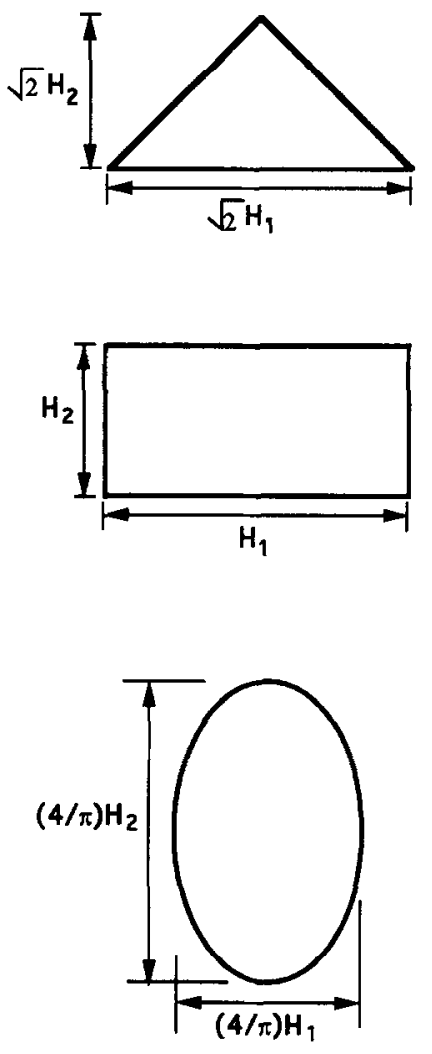

2D MODELS
SE.V $=\mathrm{K} \mathrm{H}_{1} \mathrm{H}_{2} \mathrm{H}_{3}$

$S E . S=2 \times\left\{(1 / 3)\left[\left(H_{2} H_{3}\right)^{2}+\left(H_{1} H_{3}\right)^{2}+\left(H_{1} H_{2}\right)^{2}\right]\right\}^{12}$

א

Assumed Volume

$\mathbf{K}$

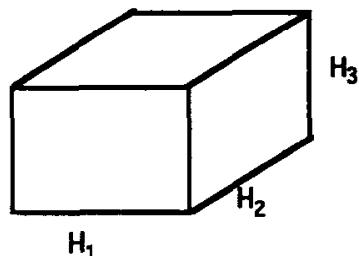

1

$(3 / 4) \mathrm{H}_{3}$

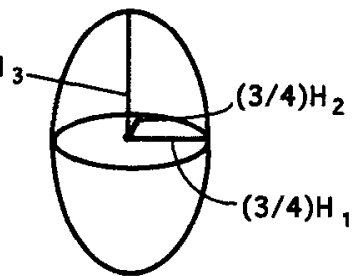

$(9 / 16) \pi$

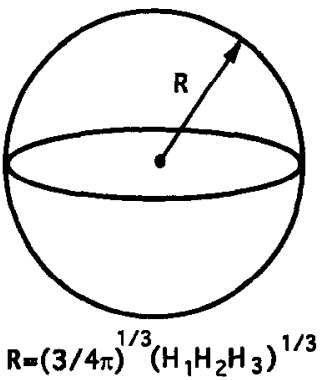

3D MODELS

Fig. 3. Three two-dimensional and three three-dimensional examples of the dependence of structural element properties (SEA, SEL, SEV, SES) upon the assumed shape of the structural area. Note that the dependencies are all identical except for the constant parameter $\kappa$.

By stereological arguments (Weibel, 1979), the three-dimensional surface-to-volume ratio of the structural element associated with this ellipsoid, SE.S/SE.V, can be estimated from the two-dimensional length to area ratio (SE.L/SE.A) as

$$
\begin{aligned}
\text { SE.S/SE.V } & \approx \frac{4}{\pi} \text { SE.L/SE.A } \\
& \approx 2\left\{\frac{1}{2}\left(\frac{1}{H_{1}^{2}}+\frac{1}{H_{2}^{2}}\right)\right\}^{1 / 2} .
\end{aligned}
$$

This approximation to the three-dimensional value is presented, since the surface area of an ellipsoid cannot be determined analytically for the same reason that the perimeter of an ellipse cannot be determined. Note that the ratio SE.S/SE.V is essentially identical to the surface-to-volume ratio BS/TV.
Recognizing that the quantity in parentheses in equation (14) is the inverse of the harmonic mean (Spiegel, 1988) of the $H_{i}^{2}, i=1,2$, it is argued that a better approximate value of SE.S/SE.V is

$$
\text { SE.S } / S E . V \approx 2\left\{\frac{1}{3}\left(\frac{1}{H_{1}^{2}}+\frac{1}{H_{2}^{2}}+\frac{1}{H_{3}^{2}}\right)\right\}^{1 / 2}
$$

where the quantity in parentheses is now the inverse of the harmonic mean in three dimensions of the squares of the principal lengths of the mean intercept ellipsoid. A more complex approximation of this ratio based on the assumption of a structural element containing an ellipsoidal hole was developed by Turner (1986).

The determination of SE.V and SE.S for the structural element follows the same argument used to define the generalized two-dimensional structural 
element. Similar to the several two-dimensional cases of Fig. 3, we propose for the three-dimensional SE,

$$
\mathrm{SE} . \mathrm{V}=\kappa \mathrm{H}_{1} \mathrm{H}_{2} \mathrm{H}_{3} \text {, }
$$

where $\kappa$ is characteristic of the specific structural element model chosen. Equations (15) and (16) immediately result in the relationship

$$
\text { SE.S } \approx 2 \kappa\left\{\frac{1}{3}\left[\left(H_{2} H_{3}\right)^{2}+\left(H_{1} H_{3}\right)^{2}+\left(H_{1} H_{2}\right)^{2}\right]\right\}^{1 / 2} \text {. }
$$

Several special cases for the structural element were considered (Fig. 3). Since each model differs only by the value of $k$ from any other, only the portions of the right-hand sides of equations (16) and (17) that depend on the $H_{i}, i=1,2,3$, were considered in the experimental examination.

\section{EXPERIMENTAL DATA}

To develop an empirical relationship between the derived measures (SE.V, SE.S and SE.S/SE.V) and the bone volume fraction (BV/TV), three-dimensional morphometric data were used. These data were measured from 107 cubic bone specimens $(8 \mathrm{~mm} / \mathrm{side})$ taken from diverse metaphyseal regions of four fresh frozen cadavers. They were scanned on a three-dimensional microcomputed tomography system at the University of Michigan (Feldkamp et al., 1989). The reconstructed micro-CT data represent the digitized three-dimensional architecture of cancellous bone using $50 \mu \mathrm{m}$ voxels (the three-dimensional equivalent of a pixel). The resulting data were then post-processed to determine the mean intercept length distribution (Goulet et al., in preparation; Kuhn et al., 1990). The data used in the present study are derived from data collected in the course of other studies and represent a reasonable range of cancellous bone microstructure, although somewhat dominated by femoral and tibial specimens (Table 1). Plots of the data used in this study are presented (Appendix 4). The range of $\mathrm{BV} / \mathrm{TV}$ was $0.06 \leqslant \mathrm{BV} / \mathrm{TV} \leqslant 0.37$

\section{EXPERIMENTAL RESULTS}

The relationship was strong between the structural element volume and the bone volume fraction, $\mathrm{SE} . \mathrm{V}=0.017 \kappa(\mathrm{BV} / \mathrm{TV})^{-2.05} \mathrm{~mm}^{3}, R^{2}=0.93$ (Fig. 4). SE.V [as calculated from equation (16)] was essentially proportional to $(\mathrm{BV} / \mathrm{TV})^{-2}$. The constant $\kappa$ is model-dependent and has no effect on the dependence on BV/TV. The relationship was determined using a linear regression on the natural $\log$ transforms of SE.V and BV/TV

The relationship between the structural element surface and bone volume fraction was also strong, SE. $S=0.144 \kappa(B V / T V)^{-1.35} \mathrm{~mm}^{2}, R^{2}=0.92$ (Fig. 5). The power of $\mathrm{BV} / \mathrm{TV}$ in the case is essentially equal to $-4 / 3$.
Table 1. The number of samples by site for the micro-CT measurement of mean intercept length $\left(H_{i}\right)$ and bone volume fraction (BV/TV)

\begin{tabular}{lrrrr}
\hline & \multicolumn{4}{c}{ Donor number } \\
\cline { 2 - 5 } Site & 3 & 8 & 9 & 10 \\
\hline R Dist. Fem. & 10 & 10 & 10 & 9 \\
R Prox. Fem. & 5 & 2 & 2 & 3 \\
R Dist. Rad. & 0 & 0 & 0 & 2 \\
L Dist. Rad. & 3 & 2 & 2 & 0 \\
R Prox. Hum. & 0 & 0 & 0 & 2 \\
L Prox. Hum. & 3 & 2 & 2 & 0 \\
R Prox. Tib. & 8 & 10 & 10 & 2 \\
L I1 Crest & 0 & 3 & 0 & 0 \\
R Il Crest & 0 & 0 & 1 & 0 \\
Lumbar Sp. & 0 & 4 & 0 & 0 \\
Donor 3 & 31-year-old male & \\
Donor 8 & 55-year-old female \\
Donor 9 & 69-year-old male \\
Donor 10 & 61-year-old female \\
\hline
\end{tabular}

The fit to a power law for the bone surface to volume ratio as a function of bone volume fraction was SE.S $/ \mathrm{SE} . \mathrm{V}=8.40(\mathrm{BV} / \mathrm{TV})^{0.705} \mathrm{~mm}^{-1}, R^{2}=0.94$ (Fig. 6). If the dependence of SE.S and SE.V were assumed to be $-4 / 3$ and -2 , respectively, then SE.S/SE.V was proportional to $(\mathrm{BV} / \mathrm{TV})^{2 / 3}$, close to the curve fit value of 0.705 .

\section{DISCUSSION}

The principal result of this work is the development of the general model and derivation of the measures of cancellous bone microstructure, SE.S, SE.V and SE.S/SE.V. The equation for SE.S/SE.V is significant because it approximates the surface-to-volume ratio of cancellous bone as a function of the $H_{i}$ without any assumptions about the trabecular microstructure. This freedom from assumption results from the direct derivation of the surface-to-volume ratio from the empirically observed ellipsoidal mean intercept distribution. The relationships for SE.V and SE.S are significant because (1) they represent measures of the volume and trabecular surface of a broad class of models for cancellous bone and (2) they are theoretically independent of the bone volume fraction. Since these derived measures are not mathematically dependent on the bone volume fraction, the high $R^{2}$ of the power-law fits found in the experimental examination point to the existence of an underlying biological mechanism for the formation and transformation of the trabecular microstructure that strictly determines the relationship between bone mass and bone microstructure. The exact nature of this mechanism is not known. However, it is certainly based on the biological dynamics of the structural transformation and, consequently, is not directly accessible to the static morphometric measures used in the current study. 


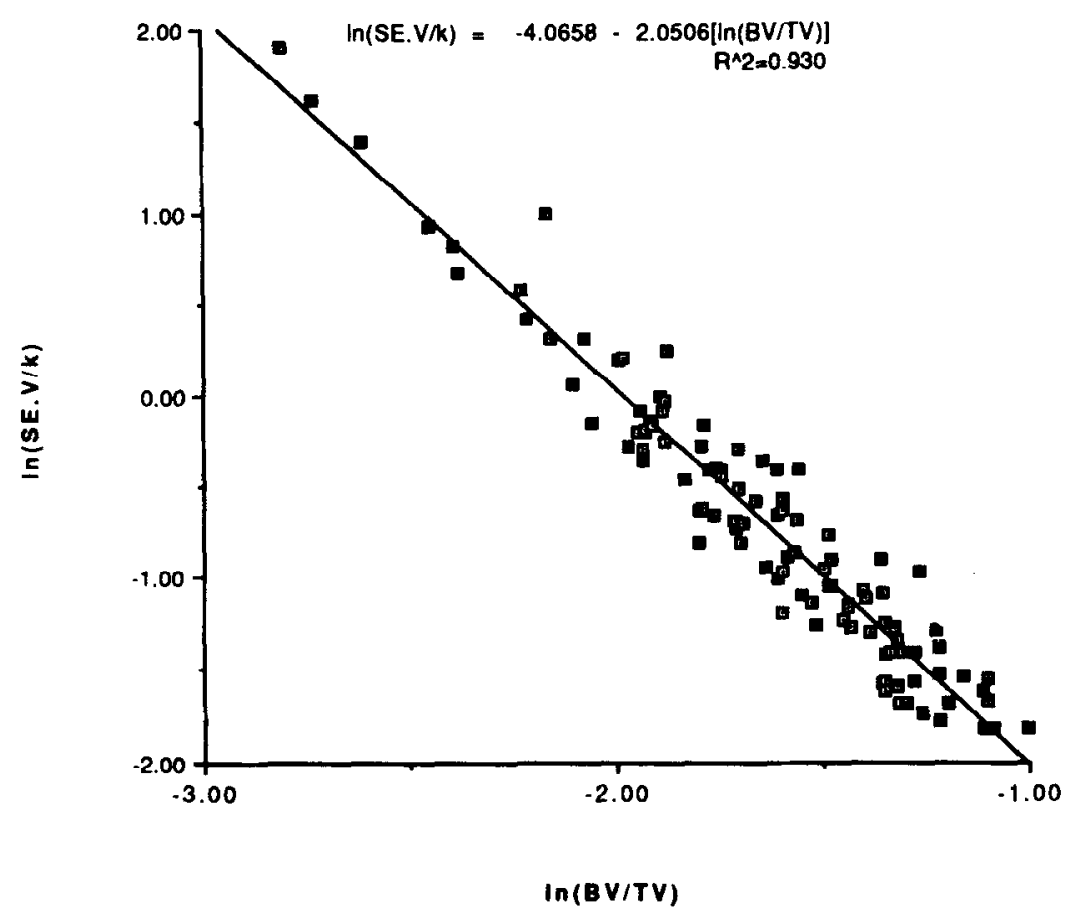

Fig. 4. Volume of the structural element (SEV) as a function of bone volume fraction (BV/TV). The best logarithmic fit had BV/TV -2.05

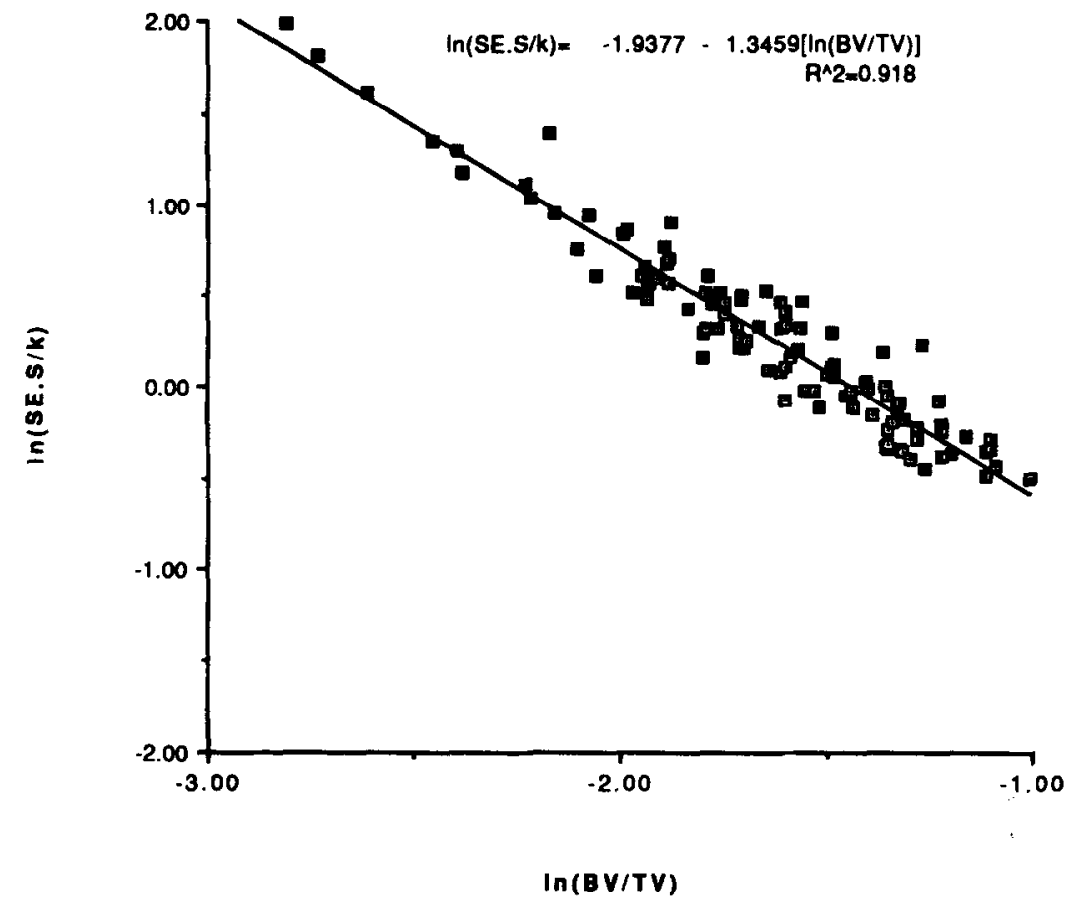

Fig. 5. Surface area of the structural element (SES) as a function of the bone volume fraction (BV/TV).

Determination of the exact nature of this dynamic mechanism and explanation of the processes which so closely relate structure and mass may require the development of novel three-dimensional dynamic morphometric techniques.
The result for the bone-marrow interface length per total tissue area, Tb.L/TA [equation (9)], is identical to the two-dimensional result of Turner (1986), who assumed that the model for bone was an elliptical (two-dimensional) or ellipsoidal (three-dimensional) 


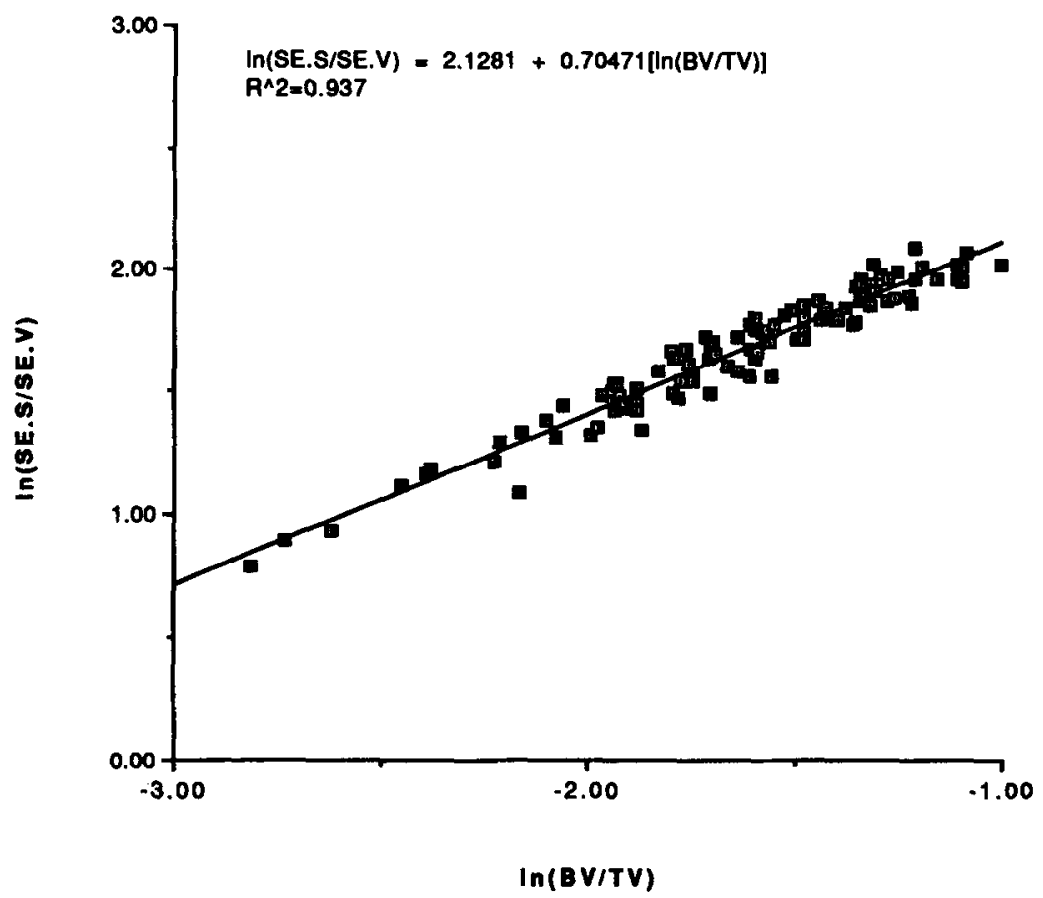

Fig. 6. Surface-to-volume ratio for the structural element (SES/SEV) plotted as a function of bone volume fraction (BV/TV). These results are similar to those of Martin (1984) for bone surface per total volume (BS/TV) as a function of bone volume fraction (BV/TV).

void in a rectangular repeated structural element. The current derivation is more general than that of Turner since no assumptions were made regarding the shape of the bone-marrow interface or the shape of the repeated area that contains the interface. This identity with the model-based result of Turner is important since he experimentally validated his equation for Tb.L/TA, demonstrating its validity for a combined sample of human and bovine cancellous bone.

Examining some of the details of these results, the experimental result for SE.V (Fig. 4) indicates that the apparent volume associated with a single trabecula is a strongly nonlinear function of BV/TV. There is a rapid increase in SE.V as BV/TV declines to zero. As a consequence of the nonlinearity of the relationship, the marrow space associated with each trabecula increases rapidly as BV/TV falls to less than 0.15 . This is consistent with a change from a more to a less crossbraced trabecular bone structure, since the removal of trabecular rods and plates simultaneously decreases BV/TV and increases the average distance between the remaining trabeculae. The trend of these results for low volume fraction is consis tent with the results of Snyder and Hayes (1990). They reported that the dependence of intertrabecular spacing on BV/TV was as the inverse of $\mathrm{BV} / \mathrm{TV}$ with an $R^{2}>0.54$ for vertebral cancellous bone and $R^{2}>0.81$ for sections from the proximal femur. This result may conflict with the experimental relationship developed here for SE.V [proportional to (BV/TV) ${ }^{-2}$ ]. It is, however, reasonable that SE.V should vary roughly as the product of the three principal intertrabecular spacings. Their curve fits result in a dependence of (BV/TV) ${ }^{3}$. To obtain the dependence of $(\mathrm{BV} / \mathrm{TV})^{-2}$ from the current study, each $H_{i}$ should be roughly proportional to (BV/TV $)^{-2 / 3}$. This difference between their curve-fit dependence for the $H_{i}$ and the dependence needed to match the SE.V curve developed here might be explained if their data were fit using a power function rather than using a linear fit. This was examined indirectly by curve fitting the $H_{i}$ data for the 107 specimens used in the current study using both methods (Appendix 4). Each approach gave such a strong relationship between the mean intercept length and BV/TV that it was unclear which might be the better approach. The dependence on BV/TV for the power-law case was approximately $-2 / 3$ for each of the $H_{i}$, consistent with the result for SEV. The rapid increase in SE.V for specimens with BV/TV less than 0.15 is consistent with their data for trabecular spacing.

The structural element surface (SE.S) decreases rapidly as BV/TV increases for all models (Fig. 5). This is consistent with the observation that the percentage bone turnover per year is much higher in cancellous bone than in cortical bone if it is assumed that the total area available for remodeling (SE.S) is a primary determinant of the rate of remodeling. This assumption is not generally accepted.

These results for SE.V as a function of BV/TV also imply a strong relationship between the volume of the structural element and bone strength. It has been 
determined experimentally that the strength of cancellous bone is roughly proportional to the square of the apparent density (Rice et al., 1988). The experimental relationship developed here for the volume of the structural element (SE.V) is inversely proportional to the square of BV/TV. Assuming that the apparent density is linearly related to BV/TV (Martin, 1984; Goulet $e t$ al., in preparation), these observations can be combined. Consequently, it is predicted that cancellous bone strength is linearly proportional to the inverse of SE.V. This is consistent with the clinical observation that dense, fine-grained cancellous bone is stronger than porous, coarse-grained bone. This relationship of structural coarseness to strength may be useful for the examination of osteoporotic bone strength and may provide further theoretical motivation for the use of texture analysis (Kaufman et al., 1988,1989 ) as a means of predicting cancellous bone strength.

The experimental results for the surface-to-volume ratio of the structural element (SE.S/SE.V) (Fig. 6) also show a strong dependence on BV/TV. This result is similar to that of Martin (1984) in which he examined the bone-marrow interface surface per total volume of cancellous bone tissue (BS/TV) for human femoral head, iliac crest, rib, vertebrae, and dog vertebrae as a function of $\mathrm{BV} / \mathrm{TV}$. His results were similar to the result of this paper for BV/TV in the range of the data used in this paper. These two approaches to the surface-to-volume ratio are essentially identical. If the number of structural elements is denoted by SE.N, then

$$
\begin{aligned}
\frac{\text { SE.S }}{\text { SE.V }} & =\frac{\text { SE.N } \times \text { SE.S }}{\text { SE.N } \times \text { SE.V }} \\
& =\frac{\text { BS }}{\mathrm{TV}} .
\end{aligned}
$$

The results for SE.S/SE.V of this paper are also similar to the results of Fazzalari et al. (1990) for BS/TV. They made analytical models for cancellous bone that assumed various microstructures and compared the model prediction of BS/TV to measurements taken from femoral head and iliac crest cancellous bone specimens. For the mathematical models the dependence of BS/TV on BV/TV was a power function with an exponent between 0.0 (plate model) and 0.67 (sphere model). Their experimental finding was that, for normal cancellous bone, the dependence of BS/TV on BV/TV had an exponent of 0.44 for the femoral head and 0.56 for the iliac crest. Differences in BS/TV versus BV/TV were expected because the structure of the SE is tightly controlled in vivo by biological processes. The biological mechanisms of trabecular formation and remodeling will determine the dependence of BS/TV on BV/TV. It is reasonable, then, that different relationships were reported for femoral and iliac crest bone (Fazzalari et al., 1990) as the cancellous bone of these regions has different structural functions. In addition to differing mechanical function, other factors modulating the relationship include disease and genetic or developmental abnormalities of bone. The exponent found in the current study $(0.70)$ is somewhat different. For Fazzalari et al.'s data, the models composed of plates and rods were better able to represent the dependence of BS/TV on BV/TV than the other models they examined. Their sphere model had a dependence of $\mathrm{BS} / \mathrm{TV}$ on BV/TV of 0.67 , quite similar to the dependence of 0.70 found in the current study. In addition, their data showed clear differences in dependence between the femoral and iliac sites. Our data could not be used to examine this difference due to the small number of iliac crest specimens.

This work shows that much additional information exists in the mean intercept ellipsoid than has heretofore been exploited directly. The major results are: (1) The theoretical estimates of the surface distribution and average volume of the basic structural element may be helpful in developing morphologically valid mechanical models for cancellous bone. (2) The strength of bone can be related to the structural element volume (texture) and (3) The empirical relationships between the derived parameters SE.V, SE.S, SE.S/SE.V and BV/TV were remarkably strong. This indicates that some underlying biological rules govern the structural element size and trabecular surface shape in cancellous bone. This is supported by the experimental results of Goldstein et al. (1990) and Snyder et al. (1990). Determination of the actual cellular mechanisms awaits collection of appropriate dynamic morphometric data.

Also made clear is that the mean intercept length measure is insufficient to completely determine the morphology of a structural element for cancellous bone. The lack of position information for the bone-marrow interface currently precludes a complete definition of the SE. Development of new morphometric measures such as the volume projection method of Reimann et al. (1992) are needed before complete morphologically accurate models for cancellous bone are possible.

Acknowledgements-The authors thank D. Reimann of the Henry Ford Hospital, Radiologic Physics and Engineering Division, for his help with MACSYMA. We also thank S. J. Hollister for reading the mathematics for content and accuracy and $T$. Wenzel for preparing the figures.

This research was supported by The Whitaker Foundation and NIH AR40776, AR34399.

\section{REFERENCES}

Cowin, S. C. (1985) The relationship between the elasticity tensor and the fabric tensor. Mech. Mater. 4, 137.

Cowin, S. C. (1989)(Editor) Bone Mechanics, Chap. 7. CRC Press, Boca Raton, FL.

Fazzalari, N. L., Crisp, D. J. and Vernon-Roberts, B. (1990) Mathematical modelling of trabecular bone structure: the evaluation of analytical and quantified surface to volume relationships in the femoral head and iliac crest. J. Biomechanics 22, 901-910. 
Feldkamp, L.A., Goldstein, S. A., Parfitt, A. M., Jesion, G. and Kleerekoper, M. (1989) The direct examination of three-dimensional bone architecture in vitro by computed tomography. J. Bone Min. Res. 4, 3-11.

Goldstein, S. A., Hollister, S. J., Kuhn, J. L. and Kikuchi, N. (1990) Biomechanics of Diarthrodial Joints, Vol. II (Edited by Mow, V. C., Ratcliffe, A. and Woo, S. L.-Y.), pp. 61-82. Springer, Berlin.

Goulet, R. W., Ciarelli, M. J., Goldstein, S. A., Kuhn, J. L., Feldkamp, L. A., Kruger, D., Viviano, D., Champlain, F. and Mathews, L. S. (1988) The effects of architecture and morphology on the mechanical properties of trabecular bone. Trans. 34th Meet. Orthop. Res. Soc., p. 73.

Goulet, R. W., Ciarelli, M. J., Goldstein, S. A., Kuhn, J. L., Feldkamp, L. A. and Brown, M. (1993) Mechanical and structural characterization of cancellous bone (Their work shows a linear relationship between apparent density and bone volume fraction with $R^{2}>0.8$ and $p<0.0005$ ) (in preparation).

Harrigan, T. P. and Mann, R. W. (1983) The mean intercept length tensor for cancellous bone. J. Biomechanics 6, 657-670.

Hilliard, J.E. (1962) Specification and measurement of microstructural anisotropy. Trans. Metallurgical Society AIME 224, 1201-1211.

Kaufman, J. J., Hakim, N., Nasser, P., Mont, M., Klion, M. Herman, G., Pilla, A. A. and Siffert, R. S. (1988) Digitil image processing of vertebral computed tomography scans for mechanical strength estimation. Trans. 34th Meet. Orthop. Res. Soc., p. 230.

Kaufman, J. J., Nasser, P., Munt, M., Hakim, N., Pilla, A. A. and Siffert, R. S. (1989) Texture analysis of vertebral computed tomography scans improve trabecular strength estimation. Trans. 35th Meet. Orthop. Res. Soc., p. 264.

Kuhn, J. L., Goldstein, S. A., Feldkamp, L. A., Goulet, R. W. and Jesion, G. (1990) Evaluation of a microcomputed tomography system to study trabecular bone architecture. J. orthop. Res. 8, 833-842.

Martin, R. B. (1984) Porosity and specific surface of bone. CRC Crit. Rev. biomed. Engng 10, 179-222.

Odgaard, A., Jensen, A. B. and Gundersen, H. J. G. (1990) Estimation of structural anisotropy using volume orientation-a new concept. Trans. 36th Meet. Orthop. Res. Soc., p. 53.

Parfitt, A. M., Drezner, M. K., Gloriex, F. H., Kanis, J. A., Malluche, H., Meunier, P. J., Ott, S. M. and Recker, R. R. (1987) Bone histomorphometry. standardization of nomenclature, symbols and units. J. Bone Min. Res. 2, 595-610.

Parfitt, A. M., Mathews, C. H. E., Villanueva, A. R., Kleerekoper, M., Frame, B. and Rao, D. S. (1983) Relationship between surface, volume and thickness of iliac trabecular bone in aging and ostcoporosis. Implications for the microanatomic and cellular mechanisms for bone loss. J. Clin. Invest. 72, 1396-1409.

Philofsky, E. M. and Hilliard, J. E. (1969) On the measurement of the orientation distribution of lineal and areal arrays. Q. appl. Math. 27, 79-86.

Raux, P., Townsend, P. R., Miegel, R., Rose, R. M. and Radin, E. L. (1975) Trabecular architecture of the human patella. J. Biomechanics 8, 1-7.

Reimann, D., Fyhrie, D. P., Fazzalari, N. and Schaffler, M. B. (1992) Trabecular bone morphometry by volume projection. Trans. 38th Meet. Orthop. Res. Soc., p. 561.

Rice, J. C., Cowin, S. C. and Bowman, J. A. (1988) On the dependence of the elasticity and strength of cancellous bone on apparent density. J. Biomechanics 21, 155-168.

Selby, S. M. (1971) (Editor) CRC Standard Mathematical Tables (19th Edn), p. 13

Snyder, B. D., Cheal, E. J., Hipp, J. A. and Hayes, W. C (1989) Anisotropic structure-property relations for trabecular bone. Trans. 35th Meet. Orthop. Res. Soc., p. 265.

Snyder, B. D. and Hayes, W. C. Biomechanics of Diarthrodial
Joints, Vol. II (Edited by Mow, V. C., Ratcliffe, A. and Woo, S. L.-Y.), pp. 31-60. Springer, Berlin.

Spiegel, M. R. (1988) Statistics, 2/ed, Shaum's Outline, p. 62. McGraw-Hill, New York.

Stone, J. L., Snyder, B. D., Hayes, W. C. and Strang, G. L. (1984) Three dimensional stress-morphology analysis of trabecular bone. Trans. 30th Ann. Meet Orthop. Res. Soc., p. 199.

Turner, C. (1986) Boundary length and internal surface area measurements in porous materials with elliptical pores. J. Mater. Sci. 21, 3907-3911.

Turner, C., Rho, J., Ashman, R. and Cowin, S. (1988) The dependence of the elastic constants of cancellous bone upon structural density and fabric. Trans. 34th Ann. Meet. Orthop. Res. Soc., p. 74.

Underwood, E. E. (1970) Quantitative Stereology. AddisonWesley, Reading, MA.

Weibel, E. R. (1979) Stereological Methods, p. 36. Academic Press, New York.

Whitehouse, W. J. (1974) The quantitative morphology of anisotropic trabecular bone. J. Microscopy 101, 153-168.

\section{APPENDIX 1 \\ USE OF MACSYMA}

The technique for performing the necessary integrations using MACSYMA is presented schematically in Fig. Al. In this figure, the sequence of commands used to calculate $y_{A}$ are presented for the first quadrant of the $X-Y$ plane. The lines labeled (c1), (c2), etc., are the commands to the program and the lines labeled (d5), (d8) are results. Note that most of the results lines were deleted for the sake of space. The commands (c1) $(\mathrm{c} 5)$ define and begin the integration of the equations. The questions after line (c5) are needed by the program for the integration. The result $(\mathrm{d} 5)$ is the original result of the integration developed by the program. The commands $(\mathrm{c} 6)-(\mathrm{c} 8)$ transform the result of $(\mathrm{d} 5)$ into the form of (d8). The final result of line (d8) is the MACSYMA form for $y_{A}$, where $h 1, h 2$ represent $H_{1}, H_{2}$ and $r$ represents the term $\theta$ from the body of this manuscript. This difference in notation arose from the way that MACSYMA represents variables. If we note that $\sin ^{2} r=1-\cos ^{2} r$ then the result (d8) can be immediately transformed to the form

$$
y_{A}=\frac{1}{2(h 2)}\left[\frac{(h 1) \cos r}{\operatorname{sqrt}\left(h 2^{2} \sin ^{2} r+h 1^{2} \cos ^{2} r\right)}-1\right], \quad 0 \leqslant r<\pi \text {. }
$$

Using a similar series of equations and transformations, the equation for $x_{A}$ is

$x_{A}=\frac{1}{2(h 1)}\left[\frac{(h 2) \sin r}{\operatorname{sqrt}\left(h 2^{2} \sin ^{2} r+h 1^{2} \cos ^{2} r\right)}\right], \quad 0 \leqslant r<\pi$.

Using these results, along with a coordinate transform [shift $y_{A}$ by $1 /(2 \times h 2)$ ], it can be immediately proven that

$$
\left[2(h 1) x_{A}\right]^{2}+\left[2(h 2) y_{A}\right]^{2}=1, \quad y_{A} \geqslant 0,
$$

which is the fundamental result used in the body of the manuscript [equation (6)]

\section{APPENDIX 2}

\section{EQUATIONS FOR STRUCTURAL ELEMENTS}

As an example of a structural element, assume that the element is an ellipse having the same Tb.L/TA as the real bone [equation (7)]. In this case the radii of the structural element (in terms of an unknown term $m$ ) are $R_{1}=m I_{1}$ and $R_{2}=m H_{2}$. The area of the ellipse is

$$
\begin{aligned}
\text { SE.A } & =\pi R_{1} R_{2} \\
& =\pi\left(m H_{1}\right)\left(m H_{2}\right) .
\end{aligned}
$$




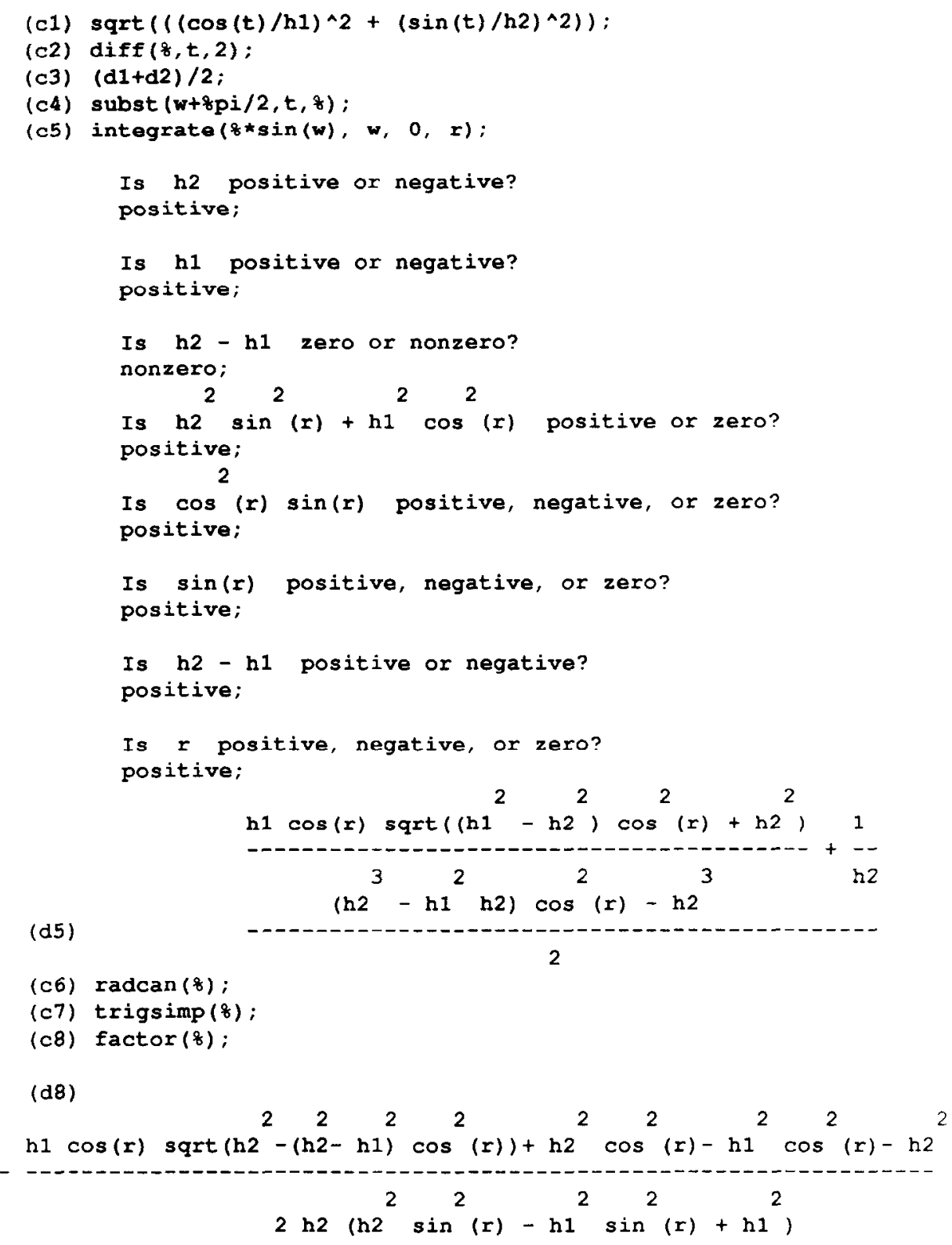

Fig. A1. Commands and edited results for calculating $y_{A}$ using MACSYMA.

By a comparison of equation (A4) with equation (10), $\kappa=m^{2} \pi$. Using $\kappa$ in the first equation (11),

$$
\text { SE.L } \approx \frac{(m \pi)^{2}}{2}\left[\left(H_{1}^{2}+H_{2}^{2}\right) / 2\right]^{1 / 2}
$$

where SE.L, SE.A are defined in the body of the manuscript.

Equations (A4), (A5), (8) and (9) together define an elliptical area that has the same mean intercept length distribution as a piece of real bone for an arbitrary magnification factor $m$. To specialize the elliptical model so that the distributed length is identical to the actual perimeter of the elliptical area, note that, for the $R_{i}$,

$$
\begin{aligned}
\text { SE. } \mathrm{L} & \approx 2 \pi\left[\left(R_{1}^{2}+R_{2}^{2}\right) / 2\right]^{1 / 2} \\
& \approx m \pi\left[\left(H_{1}^{2}+H_{2}^{2}\right) / 2\right]^{1 / 2} .
\end{aligned}
$$

Equating equations (A5) and (A6) with identification of the constant factors gives $m \pi=(\pi / 2) m^{2} \pi$. Consequently, for the special elliptical model, $m=2 / \pi$. Therefore, the equations for the structure of the elliptical structural element are

$$
\begin{aligned}
\text { SE.L } & \approx 2\left[\left(H_{1}^{2}+H_{2}^{2}\right) / 2\right]^{1 / 2}, \\
\text { SE.A } & =\frac{4}{\pi} H_{1} H_{2}, \\
\text { SE.L/SE.A } & \approx \frac{\pi}{2}\left[\frac{1}{2}\left(\frac{1}{H_{1}^{2}}+\frac{1}{H_{2}^{2}}\right)\right]^{1 / 2} .
\end{aligned}
$$

This calculation is an example of quantifying the histomorphometric properties of an assumed structural element. A selection of other assumed structural element shapes are presented in Fig. 3 with their equations for the dependence of SE.L and SE.A on $H_{i}$. 
APPENDIX 3

THE THREE-DIMENSIONAL SURFACE DISTRIBUTION ELLIPSOID

In the body of this manuscript it was argued that the three-dimensional surface distribution that has an ellipsoidal mean intercept distribution is also an ellipsoid. Following the mathematical derivation of Turner (1986), the radii of the surface distribution ellipsoid are $R_{i}=3 / 4 H_{i}$. Direct calculation of these values follows from the observation that the mean intercept length $H$ for a particular direction has the relationship to the area projection of the ellipsoid in that direction $(A)$,

$$
V=H \times A,
$$

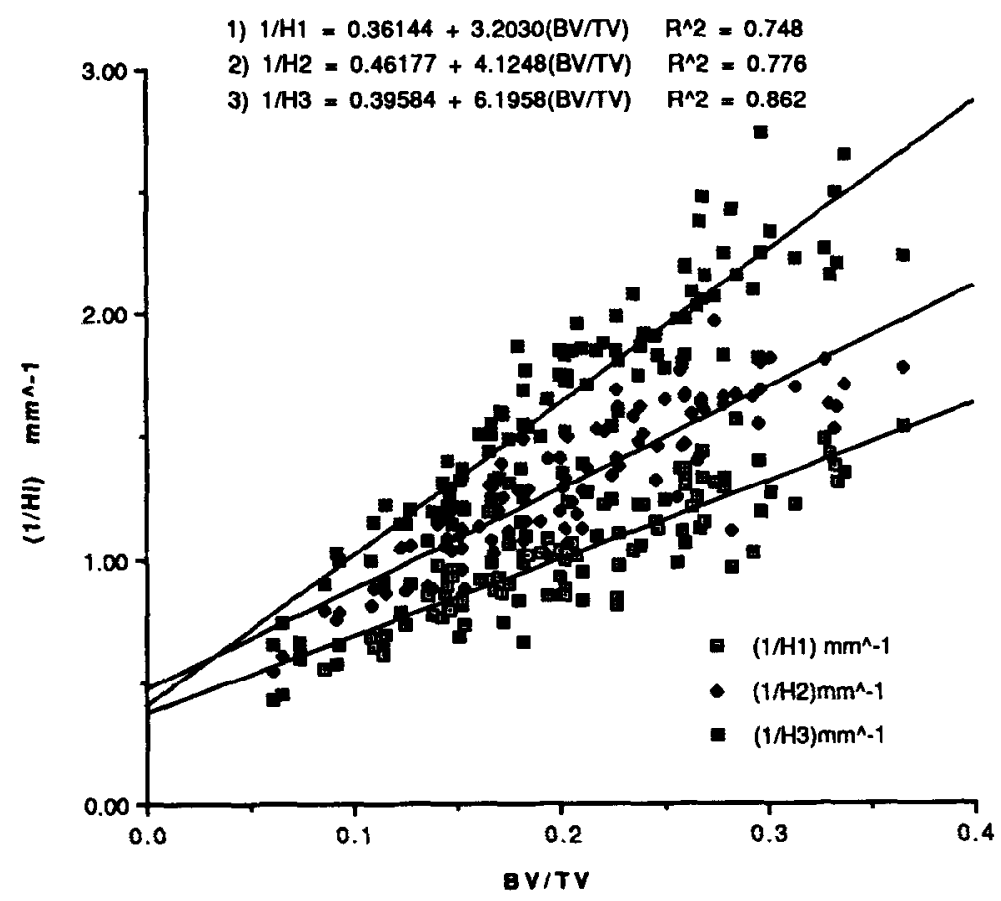

Fig. 'A2. Simple linear fit of the inverse of the principal length data $\left(H_{i}\right)$ as a function of the bone volume fraction (BV/TV).

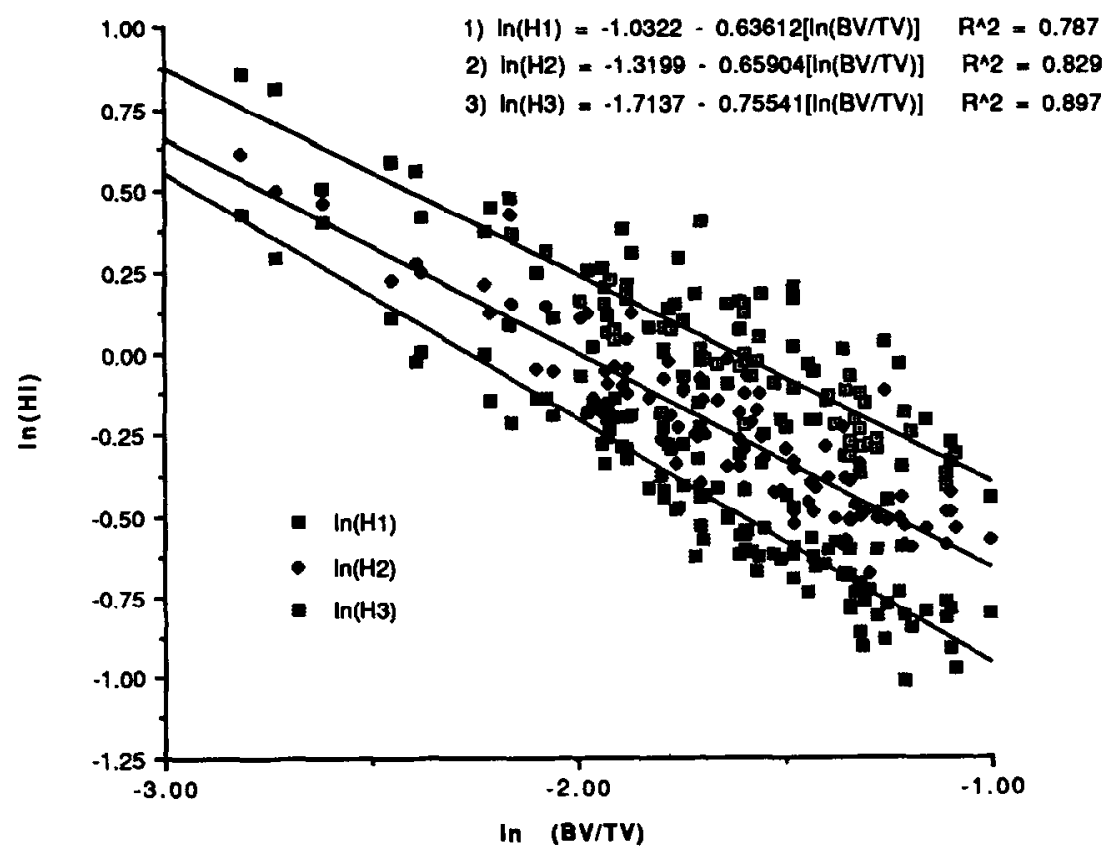

Fig. A3. Simple linear fit of the natural logarithm of the principal length data $\left(H_{i}\right)$ as a function of the natural logarithm bone volume fraction (BV/TV). 
where $V^{\prime}=(4 / 3) \pi R_{1} R_{2} R_{3}$ is the volume of the ellipsoid in terms of its three principal radii. Note that equation (A8) is an immediate consequence of the mean value theorem for integrals applied to the definition of volume for a convex body. For the "ith principal axis of the ellipsoid the projected area is $A=\pi R_{j} R_{k}$, where $R_{j}$ and $R_{k}$ are the two principal radii perpendicular to $R_{i}$. Substituting this and the expression for $V$ into equation (A8) and rearranging immediately gives $R_{i}=(3 / 4) H_{i}$

\section{APPENDIX 4}

\section{DATA USED FOR EXPERIMENTAL RESULTS}

The data used in this paper were derived from the microCT results of Goldstein and co-workers of the University of Michigan; see Kuhn et al. (1990) and Feldkamp et al. (1989) for a description of their methods. The mean intercept data as provided to the authors of the current manuscript con- forms to the equation

$$
\begin{aligned}
\frac{1}{L^{2}} & =\left(\frac{P_{l}}{2(\mathrm{BV} / \mathrm{TV})}\right)^{2} \\
& =\lambda_{1} l^{2}+\lambda_{2} m^{2}+\lambda_{3} n^{2},
\end{aligned}
$$

where $L$ is the mean intercept length, $P_{1}$ and BV/TV are as defined in the body of this paper, the quantities $\lambda_{i}, i=1,2,3$, are the coefficients of the ellipsoid that fits the experimental data for any particular bone and $l, m, n$ are direction cosines measured from the principal axes. To convert these data to the form used in this manuscript, the relationship

$$
H_{i}=\frac{1}{2(\mathrm{BV} / \mathrm{TV}) \lambda_{i}^{1 / 2}}
$$

was used.

The dependence of the $H_{i}$ on BV/TV was examined for both the simple linear fit (Fig. A2) and for the power-law fit (Fig. A3). In both cases the fit was very good. 